\title{
Acetone evaporation monitoring using a caterpillar-like microstructured fiber
}

\author{
A. D. Gomes, M. F. S. Ferreira, J. P. Moura, R. M. André, \\ S. O. Silva, et al.
}

A. D. Gomes, M. F. S. Ferreira, J. P. Moura, R. M. André, S. O. Silva, J. Kobelke, J. Bierlich, K. Wondraczek, K. Schuster, O. Frazão, "Acetone evaporation monitoring using a caterpillar-like microstructured fiber," Proc. SPIE 9634, 24th International Conference on Optical Fibre Sensors, 96342R (28 September 2015); doi: 10.1117/12.2194932

EDIE Event: International Conference on Optical Fibre Sensors (OFS24), 2015, Curitiba, Brazil 


\title{
Acetone evaporation monitoring using a caterpillar-like microstructured fiber
}

\author{
A. D. Gomes*a,b, M. F. S. Ferreira ${ }^{a, b}$, J. P. Moura ${ }^{a}$, R. M. André, ${ }^{a, b, c}$, S. O. Silva ${ }^{a}$, \\ J. Kobelke ${ }^{c}$, J. Bierlich ${ }^{c}$, K. Wondraczek ${ }^{c}$, , K. Schuster ${ }^{c}$, O. Frazão ${ }^{\text {a,b }}$ \\ ${ }^{a}$ INESC TEC and Department of Physics and Astronomy, Faculty of Sciences, University of Porto, \\ Rua do Campo Alegre 687, 4150-179 Porto, Portugal. \\ ${ }^{b}$ Dept. of Physics and Astronomy, Faculty of Sciences, University of Porto, Rua do Campo Alegre \\ 687, 4169-007 Porto, Portugal \\ ${ }^{c}$ Leibniz Institute of Photonic Technology (IPHT Jena), Albert-Einstein-Straße 9, 07745 Jena.
}

\begin{abstract}
A new microstructured optical fiber is demonstrated to detect acetone evaporation by observing the time response of the reflected signal at $1550 \mathrm{~nm}$. The sensor consists on a caterpillar-like fiber, with a transversal microfluidic channel created with a Focused Ion Beam technique, spliced to a single-mode fiber. Different stages were visible between the dipping and the evaporation of acetone and of a mixture of water and acetone. It was also possible to detect the presence of water vapor.
\end{abstract}

Keywords: Optical fiber sensor, microstructured fiber, evaporation of acetone

\section{INTRODUCTION}

The interaction between the evanescent field and fluids inserted into microstructured optical fiber holes has been the object of extensive research for sensing purposes. Further investigations have been developed using this interaction to monitor the evaporation of volatile organic compounds. Some configurations for this purpose have been recently studied, namely, with suspended-core optical fibers (SCFs) ${ }^{1}$, microstructured optical fibers (MOFs) ${ }^{2}$ and Fiber-Optic Micro-Cell Cavities. $^{3}$

The study of volatile organic compounds are very useful, mainly for industrial and environmental monitoring. In this work, a new fiber for monitoring the acetone evaporation and for steam detection is presented. The fiber is composed of a sensor head spliced to a single-mode fiber (SMF). The sensor head is a caterpillar-like MOF with microfluidic channels created using a Focused Ion Beam technique. ${ }^{4}$

\section{EXPERIMENTAL SETUP AND RESULTS}

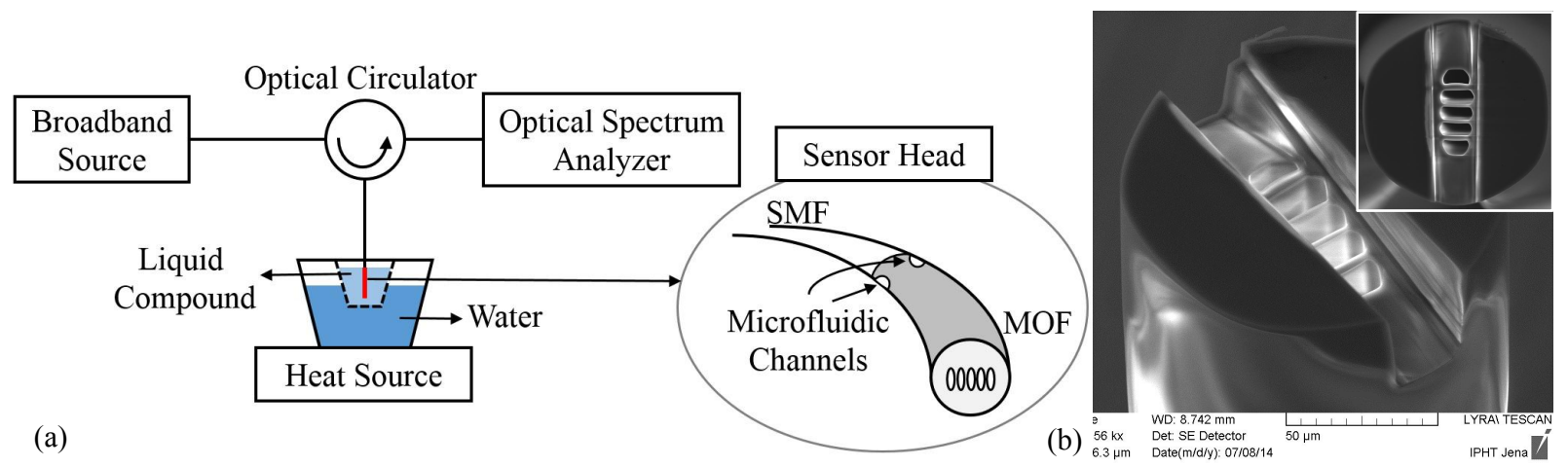

Figure 1. (a) Diagram of the experimental setup. (b) Cross section of the MOF with microfluidic channels. Image obtained by Scanning Electron Microscopy.

24th International Conference on Optical Fibre Sensors, edited by Hypolito José Kalinowski, José Luís Fabris, Wojtek J. Bock, Proc. of SPIE Vol. 9634, 96342R · (C) 2015 SPIE CCC code: $0277-786 X / 15 / \$ 18 \cdot$ doi: $10.1117 / 12.2194932$ 
The experimental setup used is shown in Figure 1(a). The setup consists in a broadband source centered at 1550nm with a bandwidth of $100 \mathrm{~nm}$ connected to an optical circulator to read the sensor response in reflection. The reflected signal was analyzed with an optical spectrum analyzer.

The sensor head is a caterpillar-like MOF with $\sim 3 \mathrm{~mm}$ length. Transversal microfluidic channels were created at one end of the fiber using a Focused Ion Beam technique and then spliced into a SMF. A cross section of the microfluidic channels is shown in Figure 1(b). This microfluidic channels allow the liquid within the MOF to flow.
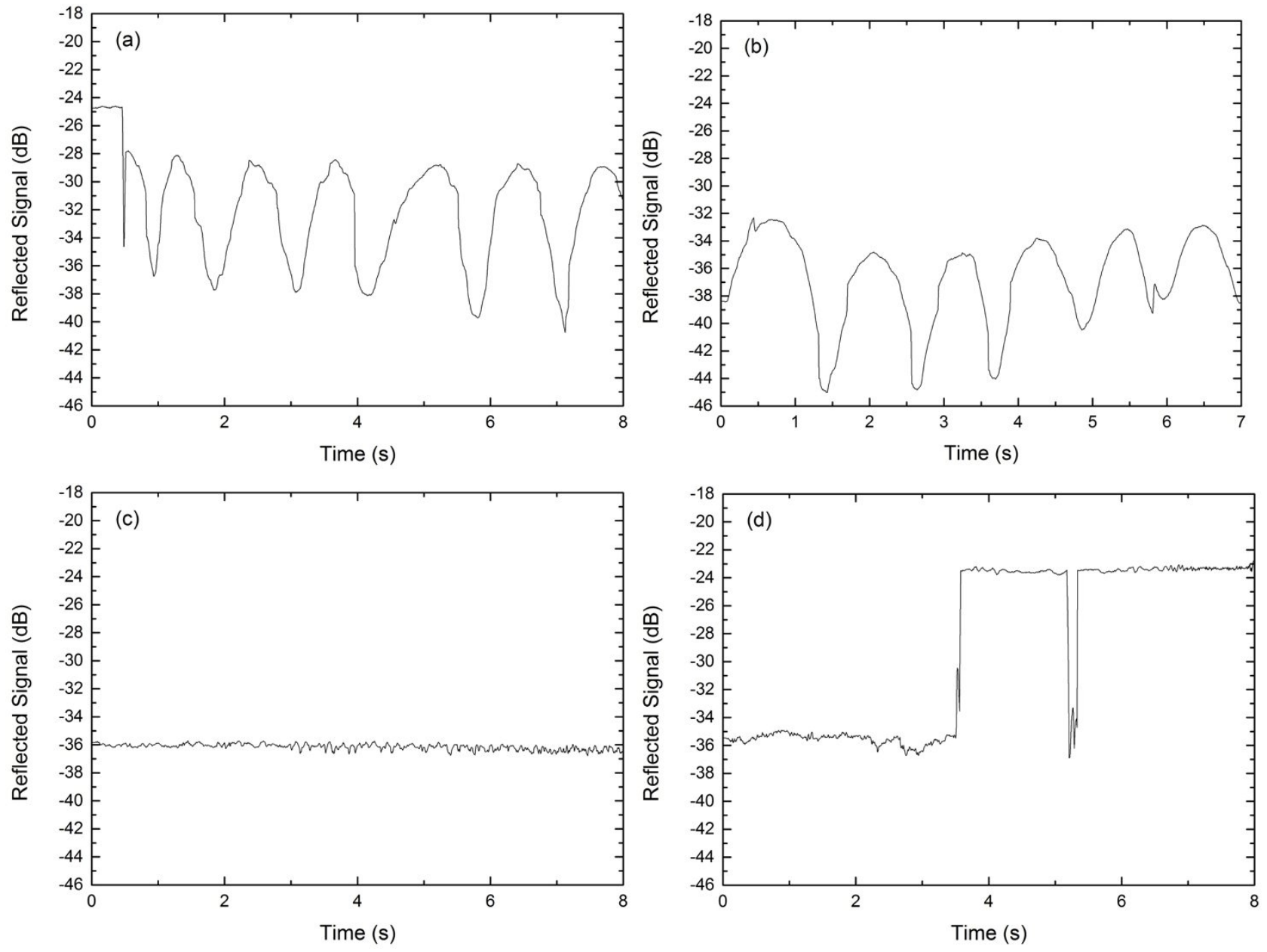

Figure 2. Time response of the reflected signal of the sensor head during the evaporation process of acetone: (a) dipping in room temperature acetone, (b) immersed in room temperature acetone, (c) immersed in acetone near the boiling point $\left(\sim 56^{\circ} \mathrm{C}\right),(\mathrm{d})$ acetone evaporation.

The sensor head was immersed in a reservoir of a liquid volatile organic compound (acetone or a mixture of $50 \%$ acetone $-50 \%$ water) at room temperature. The reservoir temperature was progressively increased using a water bath until the evaporation point of the volatile compounds $\left(\sim 56^{\circ} \mathrm{C}\right.$ for acetone $\left.e^{5}\right)$. The time response of the reflected signal from the sensor head was analyzed at $1550 \mathrm{~nm}$ since the sensor is dipped in the reservoir until the evaporation of the liquid compounds.

From this setup some problems arise. First, the liquid compound was poured manually so, depending on the speed at which the liquid is poured, the response of the sensor varies. Second, during that process, the sensor head might touch the edges of the reservoir. Consequently, perfect reproducibility during this part could not be achieved.

The response of the sensor head to the evaporation process can be divided into four different stages, represented in Figure 2. The sensor head in air, without being in contact with the liquid, has a high and stable intensity value of the reflected signal. During the dipping process in acetone at room temperature [Fig. 2(a)], the liquid enters inside the sensor 
head holes causing an increase in transmitted light. Hence, a considerable drop $(\sim 10 \mathrm{~dB})$ in the reflected signal is observed. A relatively high oscillation is observed while the sensor head is immersed in acetone at room temperature, as shown in Figure 2(a) and 2(b). During the heating process, this oscillation decreases and converges to around $-36 \mathrm{~dB}$, near the boiling point $\left(\sim 56^{\circ} \mathrm{C}\right)$ [Fig. 2(c)]. During the evaporation [Fig. 2(d)], the reflected signal starts fluctuating around $-36 \mathrm{~dB}$. When no acetone is present outside the sensor head, the air begins to fill the sensor head holes and the reflected signal rises to around $-23 \mathrm{~dB}$.

The drop in the reflected signal near 5 seconds, in Figure 2(d), might be the result of liquid acetone moving inside the sensor head until it evaporates completely. It may also be due to acetone vapor that has condensed in the sensor head, because there is still acetone to evaporate in the container bottom when there is no longer contact between the sensor head and the acetone.
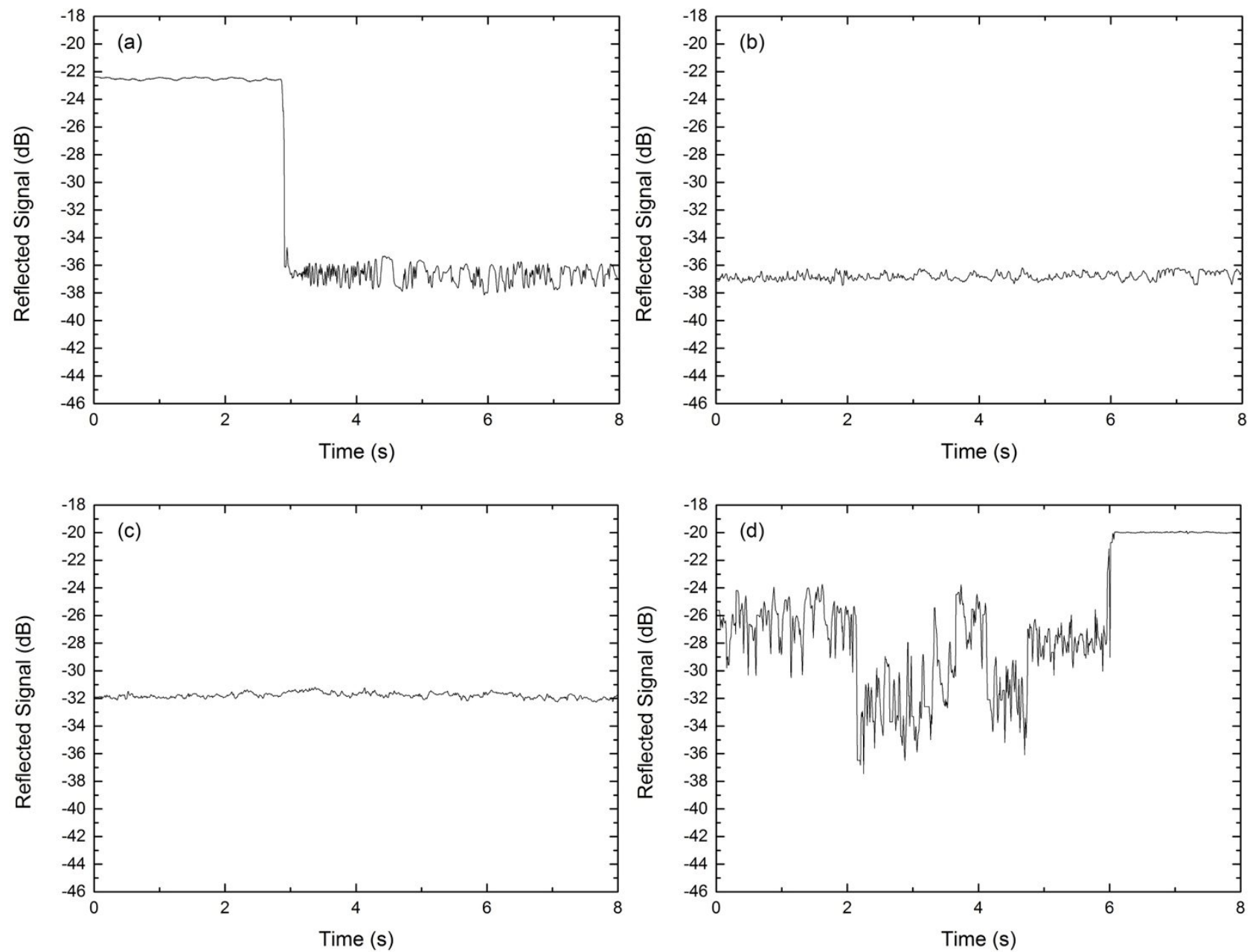

Figure 3. Time response of the reflected signal of the sensor head during the evaporation process of a $50 \%$ acetone $50 \%$ water mixture: (a) dipping in the mixture at room temperature, (b) immersed in the mixture at room temperature, (c) immersed in water, after all the acetone evaporated, (d) water evaporation.

The response from the sensor head to the evaporation process of a $50 \%$ acetone $-50 \%$ water mixture was also analyzed and can be divided into four different stages, represented in Figure 3. During the dipping process in the mixture at room temperature [Fig. 3(a)], the reflected signal decreases considerably ( 14dB), as in acetone in Figure 2(a), but is followed by a much faster oscillation with less amplitude. Shortly after dipping the sensor head into the mixture, the oscillation decreases and converges to around $-37 \mathrm{~dB}$ [Fig. 3(b)]. With increasing temperature, acetone evaporates and the sensor head remains immersed only in water. The reflected signal of the sensor head immersed in water is around $-32 \mathrm{~dB}$, as shown in Figure 3(c). As water evaporation is slow, the sensor head was manually removed from the container. 
However, there was still water within the sensor head. The sensor head was placed near the heat source so that the water in its holes could evaporate. The water evaporation can be seen in Figure 3(d). High amplitude oscillations of the reflected signal are observed right before all the water within the sensor head evaporates. The sensor was manually held, however the oscillation only started just after the sensor was near the heat source. The full evaporation of water inside the sensor head was achieved and it is represented by the rise of the reflected signal to around -20dB [Fig 3(d)].

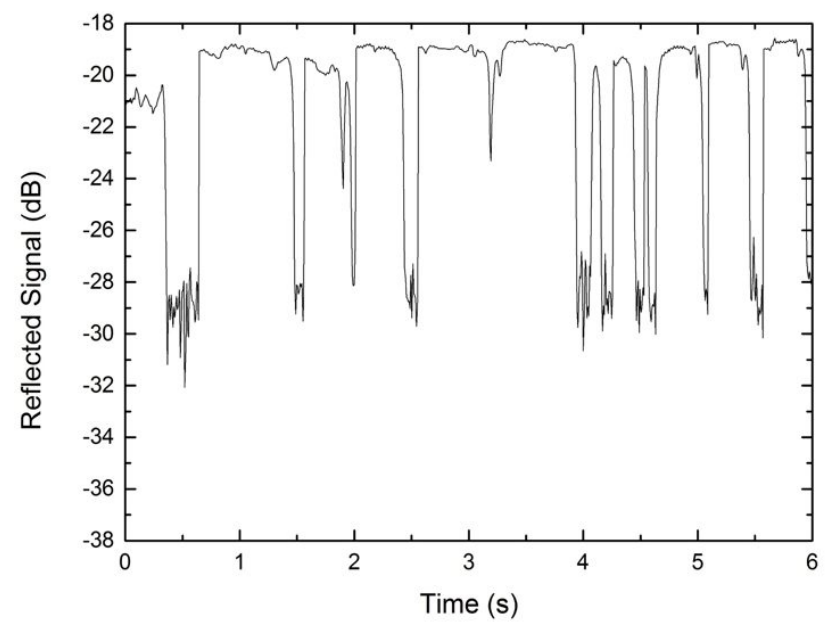

Figure 4. Time response of the reflected signal of the sensor head when exposed to water vapor.

The response of the sensor head to water vapor was also tested. The sensor head was positioned vertically above boiling water. Falls and rises from the reflected signal were observed [Fig. 4]. The steam condenses in the fiber and enters in the holes, causing more light to be transmitted and hence a drop in the reflected signal. As the water inside the fiber evaporates again, the reflected signal rises again.

\section{CONCLUSION}

The detection of acetone evaporation with the MOF dipped in acetone was achieved by observing the reflected signal at $1550 \mathrm{~nm}$. The evaporation was also detected for a $50 \%$ acetone $-50 \%$ water mixture. This detection was possible thanks to the microfluidic channels responsible for the circulation of the liquid inside the MOF. Not only that, a differentiation of several stages between the dipping and the evaporation was visible. The behavior of the signal in acetone at low temperatures differs to the one at near boiling temperatures. In the mixture, the acetone evaporation is visible with the rise of the signal's intensity. The MOF also proved to be able to detect the presence of water vapor and revealed to be very sensitive to its presence. In the future, a more detailed study of water vapor response and its condensation near the splice should be explored.

\section{ACKNOWLEDGMENTS}

Project "NORTE-07-0124-FEDER-000058" is financed by the North Portugal Regional Operational Programme (ON.2 O Novo Norte), under the National Strategic Reference Framework (NSRF), through the European Regional Development Fund (ERDF), and by national funds, through the Portuguese funding agency, Fundação para a Ciência e a Tecnologia (FCT).

\section{REFERENCES}

[1] Moura, J.P, Baierl, H., Auguste, J.-L., Jamier, R., Roy, P, Santos, J. L. and Frazão, O., "Evaporation of volatile compounds in suspended-core fibers," Opt. Lett. 39, 3868-3871 (2014)

[2] Silva, SO, Auguste, J, Jamier, R et al; "Detection of evaporation process of acetone with a microstructured fiber in a reflective configuration," Opt. Eng. 0001;53(8):080501 (2014)

[3] Preter, E., Preloznik, B., Artel, V., Sukenik, C.N., Donlagic, D., Zadok, A., "Monitoring the Evaporation of Fluids from Fiber-Optic Micro-Cell Cavities," Sensors, 13, 15261-15273 (2013).

[4] Moura, J.P., "Microstructured Optical Fibers for Fluid Sensing Applications," M.S. Dissertation, University of Porto, Faculty of Sciences, (2014).

[5] H. H. Amer, R. R. Paxton, and M. V. Winkle, “Methanol-ethanol-acetone,” Ind. Eng. Chem. 48(1), 142-146 (1956). 